\section{¿Son equivalentes los diferentes métodos para estimar la función renal en los adultos mayores?}

\author{
JORGE VEGA ${ }^{1,2}$, JUAN PABLO HUIDOBRO E. ${ }^{1,3}$, \\ RODRIGO ANDRÉS SEPÚLVEDA ${ }^{3}$
}

\section{Comparison of different methods to estimate glomerular filtration rate in older adults}

\begin{abstract}
Background: Glomerular filtration rate (GFR) estimation in older adults using a creatinine clearance/24-hour ( $\mathrm{CrCl})$ or an equation based on serum creatinine, are often considered equivalent by clinicians. Aim: To compare GFR estimated (eGFR) with the available equations and measured $\mathrm{CrCl}$ in patients $\geq 70$ years. A secondary aim was to know the differences in chronic kidney disease (CKD) classification, when using the different eGFR formulas. Patients and Methods: In 144 patients aged $77 \pm 5$ years (58\% men), $\mathrm{CrCl}$ was measured and GFR was estimated using MDRD, CKD-EPI, BIS-1 and FAS formulas. The eGFR and the stage of CKD were compared according to the different equations used. Results: GFR estimated by MDRD and CKD-EPI equations was 7.8 and $8.4 \mathrm{~mL} / \mathrm{min} / 1.73 \mathrm{~m}^{2}$ lower than 24 -hour $\mathrm{ClCr}$, respectively. This difference was even greater using the BIS-1 and FAS equations. Twenty-four-hour CrCl classified $47.2 \%$ of the patients in a category of renal function $>60 \mathrm{~mL} / \mathrm{min} / 1.73 \mathrm{~m}^{2}$, while MDRD and CKD-EPI equations classified just over a third, and BIS-1 and FAS formulas around 20\% of the patients. Conclusions: The practice of considering equivalent the GFR estimation using the creatinine-based equations with measured creatinine clearance in older adults should be reconsidered, because their results differ markedly.
\end{abstract}

(Rev Med Chile 2021; 149: 187-195)

Key words: Creatinine; Glomerular Filtration Rate; Kidney Function Tests; Renal Insufficiency, Chronic.

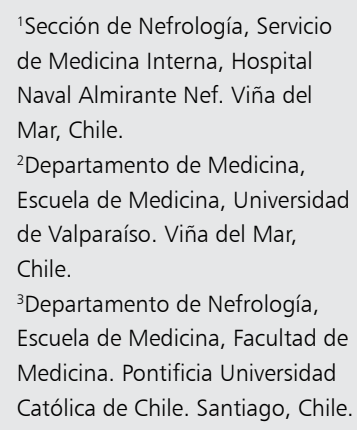

Trabajo no recibió financiamiento. Los autores declaran no tener conflictos de interés.

Recibido el 17 de agosto de 2020, aceptado 15 de diciembre de 2020 .

Correspondencia a:

Dr. Jorge Vega.

5 Norte 1035. Viña del Mar,

Chile.

jvegastieb@gmail.com
$\mathrm{F}$ recuentemente, durante la práctica clínica, en los pacientes con factores de riesgo de sufrir una enfermedad renal crónica (ERC) o en los que ya la padecen, se desea conocer el grado de función renal global, el que es inferido a través de la velocidad de filtración glomerular (VFG). Con este objetivo, habitualmente se solicita la medición de la concentración de creatinina plasmáti$\mathrm{ca}$, la que es utilizada para estimar la VFG con alguna de las fórmulas basadas en la creatinina como MDRD, CKD-EPI, BIS- 1 y FAS F $^{1-5}$. Otra alternativa para estimar la función renal es la realización de un clearance de creatinina $(\mathrm{ClCr})$, el cual puede ser medido a partir de una recolección de orina (habitualmente de $24 \mathrm{~h}$ ) o estimado con la fórmula de Cockcroft-Gault ${ }^{6}$. Sin embargo, esta conducta ha sido frecuentemente criticada en adultos mayores, porque las diferencias entre la VFG medida con métodos exactos (clearance de inulina, iohexol, iothalamato, EDTA-Cr ${ }^{51}$ y la estimada mediante los métodos anteriormente señalados son amplias. Ello puede llevar a diagnosticar una insuficiencia renal crónica inexistente cuando se subestima la VFG bajo los $60 \mathrm{~mL} / \mathrm{min} / 1,73 \mathrm{~m}^{2}$, con efectos emocionales importantes tanto en los pacientes como en sus familiares, produciendo, además, 
una sobrecarga asistencial en los policlínicos de nefrología y el gasto innecesario de recursos diagnósticos para una condición inexistente. También puede sobreestimarse la VFG, lo que puede retrasar el implementar medidas de protección renal en pacientes que podrían beneficiarse de ellas ${ }^{5,7-9}$.

Los médicos clínicos, frecuentemente, consideran al resultado de la medición del $\mathrm{ClCr} / 24 \mathrm{~h}$ similar a la estimación de VFG con alguna de las fórmulas disponibles, lo que no es correcto, especialmente en adultos mayores, ya que pueden existir diferencias significativas entre ellas.

El objetivo de este trabajo fue comparar en adultos $\geq 70$ años la evaluación de la función renal estimada con las fórmulas MDRD, CKDEPI, BIS- 1 y FAS, así como el ClCr/24 h estimado con la ecuación de Cockcroft-Gault, con el $\mathrm{ClCr}$ medido en una recolección de orina de $24 \mathrm{~h}$. El objetivo secundario fue conocer las diferencias existentes en la clasificación de los pacientes en las categorías de ERC, al utilizar los distintos métodos de estimación de la VFG.

\section{Pacientes y Métodos}

En los policlínicos de nefrología del Hospital Naval Almirante Nef, atendidos por 2 de los autores de esta investigación (J.V y J.P.H.E), se registraron en forma consecutiva, en una base de datos anonimizada durante un período de 8 meses, los datos demográficos y antropométricos (edad, sexo, raza, peso, talla, superficie corporal e índice de masa corporal [IMC]) de los pacientes $\geq 70$ años a quienes se les solicitó un $\mathrm{ClCr} / 24 \mathrm{~h}$ para la evaluación funcional de su patología renal. De este examen se registró la creatininemia, volumen urinario de $24 \mathrm{~h}$, creatininuria (en $\mathrm{mg} /$ $\mathrm{dL}$ y mg/24 h), ClCr/24 h medido y corregido por superficie corporal a $1,73 \mathrm{~m}^{2}$. Además, se registró la patología renal que motivó la solicitud de dicho examen. A esta información se agregaron los mismos parámetros obtenidos de una base de datos del Laboratorio Clínico de la Pontificia Universidad Católica de Chile recolectados entre enero de 2015 y junio de 2019. Con los datos demográficos y el valor de la creatinina sérica se estimó mediante las fórmulas CKD-EPI, MDRD, BIS-1, FAS y Cockcroft-Gault la VFG y el ClCr/24 h. Los resultados obtenidos con estas fórmulas se compararon con el $\mathrm{ClCr} / 24 \mathrm{~h}$ medido.
Se calcularon en el total de pacientes la diferencia en $\mathrm{mL} / \mathrm{min} / 1,73 \mathrm{~m}^{2}$ entre el ClCr medido y el $\mathrm{ClCr}$ estimado con la fórmula de Cockcroft-Gault y la VFG estimada (eVFG) con las fórmulas CKDEPI, MDRD, BIS-1 y FAS. Se dividió al total de pacientes en subgrupos según sexo, rango etario $(70-79$ y $\geq 80)$ e IMC $(<23,23-27,27-32$ y $>32)$ y se comparó la eVFG por los diferentes métodos para cada subgrupo.

De acuerdo con el valor obtenido del $\mathrm{ClCr} / 24 \mathrm{~h}$ (medido y estimado) y a los valores de la eVFG obtenidos con las distintas fórmulas, se los clasificó en las etapas de ERC según KDIGO.

Si un paciente tuvo más de una determinación de un $\mathrm{ClCr} / 24$ h durante el período del estudio, se consideró la primera determinación efectuada, salvo que esta haya sido omitida por cumplir con los criterios de exclusión.

El uso de las bases de datos del Hospital Naval A. Nef y de la Pontificia Universidad Católica de Chile fue autorizado por los comités ético-científicos de ambas instituciones.

La creatininemia fue procesada con un método estandarizado con IDMS y el $\mathrm{ClCr} / 24 \mathrm{~h}$ calculado con la fórmula creatininuria urinaria $(\mathrm{mg} / \mathrm{dL}) \mathrm{x}$ volumen urinario ( $\mathrm{mL} /$ minuto)/ creatininemia plasmática $(\mathrm{mg} / \mathrm{dL})$, estandarizada a una superficie corporal de $1,73 \mathrm{~m}^{2}$. La fórmula empleada para calcular la superficie corporal fue la de Dubois.

Los criterios de exclusión fueron:

1. Pacientes menores de 70 años.

2. Recolecciones inadecuadas de orina de $24 \mathrm{~h}$ de acuerdo con los requisitos establecidos por la Clínica $\mathrm{Mayo}^{10}$ (creatininurias menores de $13 \mathrm{mg} / \mathrm{kg}$ o mayores de $29 \mathrm{mg} / \mathrm{kg}$ en hombres; menores de $9 \mathrm{mg} / \mathrm{kg}$ o mayores de $26 \mathrm{mg} / \mathrm{kg}$ en mujeres; volúmenes menores de $350 \mathrm{~mL}$ o mayores de $3500 \mathrm{~mL}$ ).

3. $\mathrm{ClCr} / 24 \mathrm{~h}$ menor a $15 \mathrm{~mL} / \mathrm{min} / 1,73 \mathrm{~m}^{2}$ o estar en diálisis crónica.

Para el análisis univariado de variables cuantitativas se emplearon promedios con su respectiva desviación estándar o rango, frecuencias y porcentajes en el caso de variables categóricas. El análisis entre variables categóricas y numéricas se realizó mediante test $\mathrm{t}$ de Student, análisis de varianza y Bonferroni, dependiendo del número de categorías. Se evaluó la correlación entre los diferentes métodos de estimación de la VFG (clearance de creatinina y fórmulas) mediante análisis 
de asociación, a través de correlación lineal de Pearson y análisis de concordancia, para lo cual se utilizaron el coeficiente de correlación de Lin y gráficos de Bland-Altman. Se realizó análisis de concordancia para el diagnóstico de etapa de ERC según los diferentes métodos de estimación de la VFG mediante una matriz de coeficientes kappa y porcentajes de acuerdo.

Se consideró estadísticamente significativo un valor $\mathrm{p}<0,05$.

El análisis estadístico se realizó con el software Stata versión 14.0.

\section{Resultados}

En los 144 pacientes, la edad promedio fue $76,6 \pm 5,3$ años (rango: $70-93$ ) y $83(57,6 \%)$ fueron hombres. La creatininemia fue $1,43 \pm 0,66 \mathrm{mg} /$

Tabla 1. Características demográficas y antropométricas de 144 adultos mayores

\begin{tabular}{|lcc|}
\hline & \multicolumn{2}{c|}{ Rango } \\
\hline Edad (años), media (DS) & $76,6(5,3)$ & $70-93$ \\
\hline Sexo femenino, n (\%) & $61(42,4 \%)$ \\
\hline Peso (Kg), media (DS) & $71,2(13,7)$ & $28-105$ \\
\hline Talla (cm), media (DS) & $162,8(10,7)$ & $134-189$ \\
\hline IMC (kg/m²), media (DS) & $26,8(4,4)$ & $15,6-42,1$ \\
Superficie corporal (m²), & $1,76(0,2)$ & $1-2,2$ \\
media (DS) & & \\
\hline Creatinina (mg/dL), media (DS) & $1,43(0,66)$ & $0,6-3,67$ \\
\hline
\end{tabular}

IMC: índice de masa corporal (peso/altura²).
$\mathrm{dL} ; 1,66 \pm 0,65$ en los hombres y $1,1 \pm 0,52$ en las mujeres (Tabla 1).

En la población de adultos mayores utilizada en este estudio, el $\mathrm{ClCr} / 24 \mathrm{~h}$ no corregido fue $60,6 \pm 26,8 \mathrm{~mL} / \mathrm{min}$ y el ajustado a una superficie corporal estándar $59,6 \pm 25,4 \mathrm{~mL} / \mathrm{min} / 1,73 \mathrm{~m}^{2}$. La ecuación de Cockcroft-Gault (no estandarizada por superficie corporal) estimó el $\mathrm{ClCr} / 24 \mathrm{~h}$ en $12,3 \mathrm{~mL} / \mathrm{min}$ menos que el valor medido. $\mathrm{Al}$ utilizar las ecuaciones MDRD y CKD-EPI para estimar la VFG los valores fueron inferiores en 7,8 y $8,4 \mathrm{~mL} / \mathrm{min} / 1,73 \mathrm{~m}^{2}$, respectivamente, respecto al $\mathrm{ClCr} / 24 \mathrm{~h}$ medido. La diferencia fue aun mayor al utilizar las fórmulas BIS-1 y FAS, las que estimaron la VFG en 12,5 y $13,2 \mathrm{~mL} / \mathrm{min} / 1,73 \mathrm{~m}^{2}$ menos que el $\mathrm{ClCr} / 24 \mathrm{~h}$ medido, respectivamente (Figura 1 y Tabla 2).

En el análisis de subgrupos, la estimación de la VFG por todos los métodos ajustados por superficie corporal fue significativamente mayor en mujeres que en hombres $(\mathrm{p}<0,05)$. La evaluación de la función renal por $\mathrm{ClCr}$ no corregido y fórmula de Cockroft-Gault no indexada a superficie corporal, en tanto, no tuvo diferencias significativas entre hombres y mujeres (Tabla 2). La función renal estimada por las fórmulas de Cockroft-Gault (tanto no ajustada como indexada), BIS-1 y FAS fue mayor en el grupo de pacientes entre 70-79 años que en el grupo de 80 años o más; en cambio, al ser determinada por $\mathrm{ClCr}$ o $\mathrm{ClCr}$ corregido o estimada con las fórmulas MDRD y CKD-EPI, no mostró diferencias significativas (Tabla 2). Al analizar la población por IMC, los grupos con sobrepeso y obesidad tuvieron una función renal significativamente mayor a los grupos normo y bajo peso

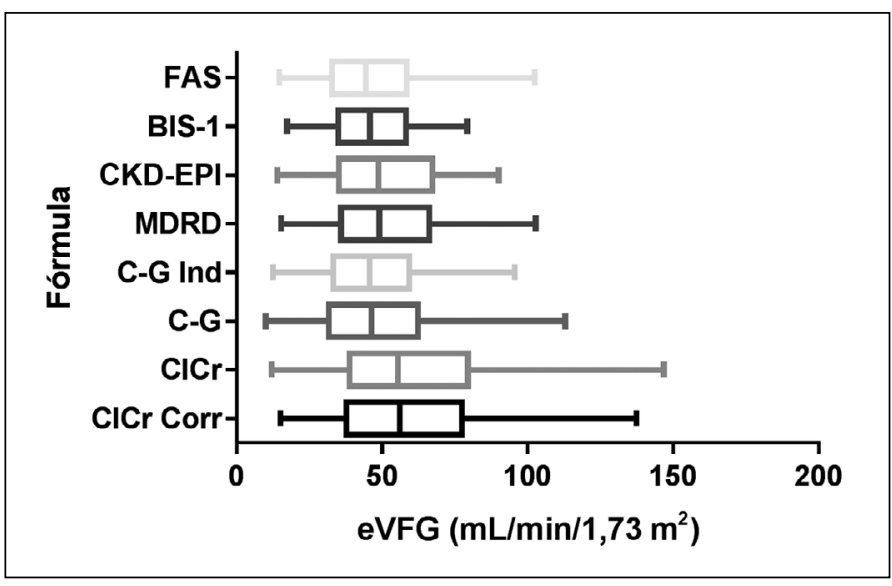

Figura 1. Rango y dispersión de los métodos para estimar la VFG en adultos mayores. $\mathrm{Cl} \mathrm{Cr}$ : Clearance de creatinina de $24 \mathrm{~h} ; \mathrm{Cl} \mathrm{Cr}$ corr: Clearance de creatinina de $24 \mathrm{~h}$ normalizado a superficie corporal de 1,73 $\mathrm{m}^{2}$; C-G: Cockroft-Gault; C-G Ind: Cockroft-Gault normalizada a superficie corporal de 1,73 m²; MDRD: Modification of diet in renal disease; CKD-EPI: Chronic Kidney Disease Epidemiology Collaboration; BIS-1: Berlin Iniciative Study-1; FAS: Full-Age Spectrum. eVFG: estimación de la velocidad de filtración glomerular. 
Tabla 2. VFG determinada por diferentes métodos en adultos mayores

\begin{tabular}{|c|c|c|c|c|c|c|c|c|c|}
\hline & & $\mathrm{Cl} \mathrm{Cr}$ & $\begin{array}{c}\mathrm{Cl} \mathbf{C r} \\
\text { corregido }\end{array}$ & C-G & $\begin{array}{c}\text { C-G } \\
\text { indexado }\end{array}$ & MDRD & CKD-EPI & BIS-1 & FAS \\
\hline \multicolumn{2}{|c|}{ Población total media (DS) } & $\begin{array}{c}60,6 \\
(26,8)\end{array}$ & $\begin{array}{c}59,6 \\
(25,4)\end{array}$ & $\begin{array}{c}48,3 \\
(19,9)\end{array}$ & $\begin{array}{c}47,7 \\
(19,0)\end{array}$ & $\begin{array}{c}51,8 \\
(21,0)\end{array}$ & $\begin{array}{c}51,2 \\
(21,0)\end{array}$ & $\begin{array}{r}47,1 \\
(15,4)\end{array}$ & $\begin{array}{c}46,4 \\
(17,3)\end{array}$ \\
\hline \multicolumn{10}{|l|}{ Sexo } \\
\hline Masculino & $\begin{array}{l}(n=83) \\
\text { media (DS) }\end{array}$ & $\begin{array}{c}60,8 \\
(27,3)\end{array}$ & $\begin{array}{l}55,8^{*} \\
(24,1)\end{array}$ & $\begin{array}{c}46,6 \\
(18,7)\end{array}$ & $\begin{array}{c}42,7^{*} \\
(16,0)\end{array}$ & $\begin{array}{c}47,5^{*} \\
(19,2)\end{array}$ & $\begin{array}{c}46,4^{*} \\
(18,9)\end{array}$ & $\begin{array}{c}43,6^{*} \\
(13,6)\end{array}$ & $\begin{array}{l}42,7^{*} \\
(15,2)\end{array}$ \\
\hline Femenino & $\begin{array}{l}(\mathrm{n}=61) \\
\text { media (DS) }\end{array}$ & $\begin{array}{c}60,4 \\
(26,4)\end{array}$ & $\begin{array}{l}64,8^{*} \\
(26,3)\end{array}$ & $\begin{array}{c}50,7 \\
(21,4)\end{array}$ & $\begin{array}{l}54,1^{*} \\
(21,1)\end{array}$ & $\begin{array}{c}57,5^{*} \\
(22,0)\end{array}$ & $\begin{array}{l}57,8^{*} \\
(22,1)\end{array}$ & $\begin{array}{c}51,9^{*} \\
(16,5)\end{array}$ & $\begin{array}{r}51,4^{*} \\
(18,9)\end{array}$ \\
\hline \multicolumn{10}{|l|}{ IMC } \\
\hline$<23 \mathrm{~kg} / \mathrm{m}^{2}$ & $\begin{array}{l}\mathrm{n}=25(17,4 \%) \\
\text { media (DS) }\end{array}$ & $\begin{array}{c}43,2^{*} \\
(18,8)\end{array}$ & $\begin{array}{l}49,0^{*} \\
(21,8)\end{array}$ & $\begin{array}{c}32,3^{*} \\
(12,8)\end{array}$ & $\begin{array}{c}36,7^{*} \\
(15,1)\end{array}$ & $\begin{array}{c}46,7 \\
(20,9)\end{array}$ & $\begin{array}{c}46,1 \\
(21,1)\end{array}$ & $\begin{array}{c}42,9 \\
(15,9)\end{array}$ & $\begin{array}{c}41,6 \\
(17,2)\end{array}$ \\
\hline $23-27,9 \mathrm{~kg} / \mathrm{m}^{2}$ & $\begin{array}{l}\mathrm{n}=64(44,4 \%) \\
\text { media (DS) }\end{array}$ & $\begin{array}{l}59,1^{*} \\
(25,3)\end{array}$ & $\begin{array}{l}57,7^{*} \\
(24,4)\end{array}$ & $\begin{array}{c}46,3^{*} \\
(17,0)\end{array}$ & $\begin{array}{c}45,5^{*} \\
(17,5)\end{array}$ & $\begin{array}{c}51,4 \\
(20,8)\end{array}$ & $\begin{array}{c}50,6 \\
(20,8)\end{array}$ & $\begin{array}{c}46,8 \\
(15,3)\end{array}$ & $\begin{array}{c}46,3 \\
(17,8)\end{array}$ \\
\hline $28-31,9 \mathrm{~kg} / \mathrm{m}^{2}$ & $\begin{array}{l}\mathrm{n}=36(25,0 \%) \\
\text { media (DS) }\end{array}$ & $\begin{array}{l}65,6^{*} \\
(25,8)\end{array}$ & $\begin{array}{c}61,4^{*} \\
(24,6)\end{array}$ & $\begin{array}{c}53,7^{*} \\
(17,4)\end{array}$ & $\begin{array}{c}50,3^{*} \\
(17,8)\end{array}$ & $\begin{array}{c}51,9 \\
(20,9)\end{array}$ & $\begin{array}{c}51,5 \\
(21,1)\end{array}$ & $\begin{array}{c}47,2 \\
(14,9)\end{array}$ & $\begin{array}{c}46,4 \\
(16,3)\end{array}$ \\
\hline$>32 \mathrm{~kg} / \mathrm{m}^{2}$ & $\begin{array}{l}\mathrm{n}=19(13,2 \%) \\
\text { media (DS) }\end{array}$ & $\begin{array}{c}79,2^{*} \\
(29,2)\end{array}$ & $\begin{array}{c}76,4^{*} \\
(27,4)\end{array}$ & $\begin{array}{c}66,0 * \\
(24,0)\end{array}$ & $\begin{array}{c}63,2^{*} \\
(21,2)\end{array}$ & $\begin{array}{c}59,2 \\
(21,3)\end{array}$ & $\begin{array}{c}59,5 \\
(21,0)\end{array}$ & $\begin{array}{c}53,6 \\
(15,4)\end{array}$ & $\begin{array}{c}53,0 \\
(17,1)\end{array}$ \\
\hline \multicolumn{10}{|l|}{ Rango etario } \\
\hline 70-79 años & $\begin{array}{l}(\mathrm{n}=105) \\
\text { media (DS) }\end{array}$ & $\begin{array}{c}63,0 \\
(26,7)\end{array}$ & $\begin{array}{c}61,5 \\
(25,4)\end{array}$ & $\begin{array}{c}51,7 * \\
(19,7)\end{array}$ & $\begin{array}{c}50,5^{*} \\
(19,0)\end{array}$ & $\begin{array}{c}52,9 \\
(20,9)\end{array}$ & $\begin{array}{c}52,9 \\
(21,0)\end{array}$ & $\begin{array}{c}49,1^{*} \\
(15,4)\end{array}$ & $\begin{array}{r}48,5^{*} \\
(17,5)\end{array}$ \\
\hline 80 o más años & $\begin{array}{l}(\mathrm{n}=39) \\
\text { media (DS) }\end{array}$ & $\begin{array}{c}54,2 \\
(26,4)\end{array}$ & $\begin{array}{c}54,4 \\
(24,9)\end{array}$ & $\begin{array}{c}39,3^{*} \\
(17,7)\end{array}$ & $\begin{array}{c}39,6^{*} \\
(17,2)\end{array}$ & $\begin{array}{c}48,6 \\
(21,1)\end{array}$ & $\begin{array}{c}46,6 \\
(20,6)\end{array}$ & $\begin{array}{c}41,6^{*} \\
(14,2)\end{array}$ & $\begin{array}{r}40,7^{*} \\
(15,8)\end{array}$ \\
\hline
\end{tabular}

$\mathrm{Cl}$ Cr: Clearance de creatinina de $24 \mathrm{~h} ; \mathrm{Cl} \mathrm{Cr}$ corregido: Clearance de creatinina de $24 \mathrm{~h}$ normalizado a superficie corporal de 1,73 m²; C-G: Cockroft-Gault; C-G indexado: Cockroft-Gault normalizado a superficie corporal de 1,73 m²; MDRD: Modification of diet in renal disease; CKD-EPI: Chronic Kidney Disease Epidemiology Collaboration; BIS-1: Berlin Iniciative Study-1; FAS: FuII-Age Spectrum; IMC: Índice de masa corporal; DS: Desviación estándar. *Diferencias significativas $(p<0,05)$ entre subgrupos.

cuando esta se estimó por $\mathrm{ClCr}, \mathrm{ClCr}$ corregido y fórmula de Cockroft-Gault $(\mathrm{p}<0,05)$; en cambio, cuando se estimó por fórmulas MDRD, CKD-EPI, BIS-1 y FAS, no hubo diferencias significativas entre los diferentes rangos de IMC (Tabla 2).

La superficie corporal fue significativamente mayor en hombres que en mujeres y en grupos de IMC mayores ( $\mathrm{p}<0,05$ para todos los subgrupos).

El ClCr/24 h medido clasificó a cerca de la mitad de los pacientes $(47,2 \%)$ en una categoría de función renal $\geq 60 \mathrm{~mL} / \mathrm{min} / 1,73 \mathrm{~m}^{2}$ (correspondiente a las etapas 1-2 de ERC). Sin embargo, la estimación de este parámetro empleando la fórmula de Cockcroft-Gault indexada puso en esta categoría solo a $24,3 \%$ de los pacientes. Las ecuaciones de MDRD y CKD-EPI clasificaron a poco más de un tercio de los pacientes en esta categoría y las fórmulas BIS-1 y FAS a alrededor de $20 \%$ de los enfermos (Tabla 3 ).

Las ecuaciones que más frecuentemente clasificaron a los pacientes en las categorías de función renal entre 30 y $59 \mathrm{~mL} / \mathrm{min} / 1,73 \mathrm{~m}^{2}$ (correspondiente a la etapa 3 de ERC) fueron BIS-1, FAS y Cockcroft-Gault indexada (54,9 a 65,3\%). El ClCr/24 h medido clasificó en estas categorías solo a $41,0 \%$ de los pacientes (Tabla 3 ).

El ClCr/24 h medido clasificó a 11,8\% de los pacientes en la categoría de función renal entre 15 y $29 \mathrm{~mL} / \mathrm{min} / 1,73 \mathrm{~m}^{2}$ (correspondiente a la etapa 4 de ERC). Las ecuaciones de Cockroft-Gault y FAS, en cambio, clasificaron en esta categoría a cerca de $20 \%$ de los pacientes (Tabla 3 ).

La concordancia entre los diferentes métodos de estimación de la función renal para clasificar a los pacientes en etapas de ERC fue disímil: entre 
Tabla 3. Clasificación en las etapas de enfermedad renal crónica según los diferentes métodos empleados en la determinación de la VFG

\begin{tabular}{|c|c|c|c|c|c|c|c|c|}
\hline $\begin{array}{l}\text { Etapa } \\
\text { ERC }\end{array}$ & $\mathrm{Cl} \mathrm{Cr}$ & $\begin{array}{c}\mathrm{Cl} \mathrm{Cr} \\
\text { corregido }\end{array}$ & C-G & $\begin{array}{c}\text { C-G } \\
\text { indexado }\end{array}$ & MDRD & CKD-EPI & BIS-1 & FAS \\
\hline $1-2$ & $66(45,8 \%)$ & $68(47,2 \%)$ & $40(27,8 \%)$ & $35(24,3 \%)$ & $52(36,1 \%)$ & $52(36,1 \%)$ & $29(20,1 \%)$ & $30(20,8 \%)$ \\
\hline 3 & $62(43,1 \%)$ & $59(41,0 \%)$ & $73(50,7 \%)$ & 79 (54,9\%) & $70(48,6 \%)$ & $66(45,8 \%)$ & $94(65,3 \%)$ & $85(59,0 \%)$ \\
\hline 4 & 15 (10,4\%) & $17(11,8 \%)$ & $28(19,4 \%)$ & $29(20,1 \%)$ & $22(15,3 \%)$ & $25(17,4 \%)$ & $21(14,6 \%)$ & $28(19,5 \%)$ \\
\hline 5 & $1 \quad(0,7 \%)$ & 0 & $3(2,1 \%)$ & $1 \quad(0,7 \%)$ & 0 & $1 \quad(0,7 \%)$ & 0 & $1 \quad(0,7 \%)$ \\
\hline
\end{tabular}

ERC: Enfermedad renal crónica; VFG: Velocidad de filtración glomerular; ClCr: Clearance de creatinina de 24 h; $\mathrm{ClCr}$ corregido: Clearance de creatinina de 24 h normalizado a superficie corporal de 1,73 m²; C-G: Cockroft-Gault; C-G indexado: Cockroft-Gault normalizado a superficie corporal de 1,73 m²; MDRD: Modification of diet in renal disease; CKD-EPI: Chronic Kidney Disease Epidemiology Collaboration; BIS-1: Berlin Iniciative Study-1; FAS: Full-Age Spectrum.

las fórmulas MDRD y CKD-EPI, y entre BIS-1 y FAS fue casi perfecta; mientras que la relación de la fórmula Cockroft-Gault con el resto de las ecuaciones fue solo moderada. MDRD tuvo una concordancia moderada con BIS-1 y FAS, mientras que CKD-EPI tuvo una concordancia mayor. La concordancia entre el $\mathrm{ClCr} / 24 \mathrm{~h}$ con las fórmulas fue entre ligera a moderada (Tabla 4).

La correlación lineal (Pearson) para estimar la VFG fue muy alta entre todos los métodos, particularmente entre las ecuaciones Cockroft-Gault indexada, MDRD, CKD-EPI, BIS-1 y FAS. La concordancia, evaluada por coeficiente de concordancia de Lin, fue muy alta entre las ecuaciones

Tabla 4. Matriz de coeficientes kappa y porcentaje de concordancia con la etapa de ERC para las distintas formas de determinación de la VFG en adultos mayores

\begin{tabular}{|c|c|c|c|c|c|c|c|}
\hline & $\begin{array}{c}\mathrm{Cl} \mathrm{Cr} \\
\text { corregido }\end{array}$ & C-G & $\begin{array}{c}\text { C-G } \\
\text { indexado }\end{array}$ & MDRD & CKD-EPI & BIS-1 & FAS \\
\hline $\mathrm{Cl} \mathrm{Cr}$ & $\begin{array}{c}0,76 \\
83,33 \%\end{array}$ & $\begin{array}{c}0,36 \\
56,25 \%\end{array}$ & $\begin{array}{c}0,28 \\
51,39 \%\end{array}$ & $\begin{array}{c}0,36 \\
56,25 \%\end{array}$ & $\begin{array}{c}0,35 \\
55,56 \%\end{array}$ & $\begin{array}{c}0,27 \\
52,78 \%\end{array}$ & $\begin{array}{c}0,23 \\
48,61 \%\end{array}$ \\
\hline $\begin{array}{l}\mathrm{Cl} \mathrm{Cr} \\
\text { corregido }\end{array}$ & & $\begin{array}{c}0,33 \\
54,17 \%\end{array}$ & $\begin{array}{c}0,34 \\
55,56 \%\end{array}$ & $\begin{array}{c}0,48 \\
65,28 \%\end{array}$ & $\begin{array}{c}0,44 \\
62,50 \%\end{array}$ & $\begin{array}{c}0,33 \\
56,94 \%\end{array}$ & $\begin{array}{c}0,28 \\
52,08 \%\end{array}$ \\
\hline C-G & & & $\begin{array}{c}0,73 \\
83,33 \%\end{array}$ & $\begin{array}{c}0,48 \\
65,97 \%\end{array}$ & $\begin{array}{c}0,57 \\
72,22 \%\end{array}$ & $\begin{array}{c}0,59 \\
75,69 \%\end{array}$ & $\begin{array}{c}0,63 \\
77,08 \%\end{array}$ \\
\hline $\begin{array}{l}\text { C-G } \\
\text { indexado }\end{array}$ & & & & $\begin{array}{c}0,54 \\
70,83 \%\end{array}$ & $\begin{array}{c}0,67 \\
79,17 \%\end{array}$ & $\begin{array}{c}0,71 \\
83,33 \%\end{array}$ & $\begin{array}{c}0,78 \\
86,81 \%\end{array}$ \\
\hline MDRD & & & & & $\begin{array}{c}0,85 \\
90,28 \%\end{array}$ & $\begin{array}{c}0,56 \\
73,61 \%\end{array}$ & $\begin{array}{c}0,57 \\
72,92 \%\end{array}$ \\
\hline CKD-EPI & & & & & & $\begin{array}{c}0,66 \\
79,17 \%\end{array}$ & $\begin{array}{c}0,70 \\
81,25 \%\end{array}$ \\
\hline BIS-1 & & & & & & & $\begin{array}{c}0,86 \\
92,36 \%\end{array}$ \\
\hline
\end{tabular}

Se muestra la concordancia en la categorización en etapas de ERC por los diferentes métodos de estimación de la VFG a través del coeficiente kappa (interpretación abajo) y el porcentaje de observaciones que son incluidas en la misma etapa de ERC por los diferentes métodos.

\begin{tabular}{|c|c|}
\hline Coeficiente & Interpretación \\
$<0,00$ & Pobre \\
0,00 a 0,40 & Ligero \\
0,41 a 0,80 & Moderado \\
0,81 a 1,00 & Casi perfecto \\
\hline
\end{tabular}

$\mathrm{Cl}$ Cr: Clearance de creatinina de $24 \mathrm{~h}$; $\mathrm{Cl} \mathrm{Cr}$ corregido: Clearance de creatinina de $24 \mathrm{~h}$ normalizado a superficie corporal de 1,73 $\mathrm{m}^{2}$; C-G: Cockroft-Gault; C-G indexado: Cockroft-Gault normalizado a superficie corporal de 1,73 m²; MDRD: Modification of diet in renal disease; CKD-EPI: Chronic Kidney Disease Epidemiology Collaboration; BIS-1: Berlin Iniciative Study-1; FAS: Full-Age Spectrum. 
Tabla 5. Matriz de correlación lineal y concordancia entre los diferentes métodos de estimación de la VFG en adultos mayores

\begin{tabular}{|c|c|c|c|c|c|c|c|}
\hline & $\begin{array}{c}\mathrm{Cl} \mathrm{Cr} \\
\text { corregido }\end{array}$ & C-G & $\begin{array}{c}\text { C-G } \\
\text { indexado }\end{array}$ & MDRD & CKD-EPI & BIS-1 & FAS \\
\hline $\mathrm{Cl} \mathrm{Cr}$ & $\begin{array}{l}0,97 \\
0,96\end{array}$ & $\begin{array}{l}0,88 \\
0,74\end{array}$ & $\begin{array}{l}0,83 \\
0,66\end{array}$ & $\begin{array}{l}0,78 \\
0,71\end{array}$ & $\begin{array}{l}0,79 \\
0,71\end{array}$ & $\begin{array}{l}0,79 \\
0,57\end{array}$ & $\begin{array}{l}0,78 \\
0,59\end{array}$ \\
\hline $\begin{array}{l}\mathrm{Cl} \mathrm{Cr} \\
\text { corregido }\end{array}$ & & $\begin{array}{l}0,84 \\
0,73\end{array}$ & $\begin{array}{l}0,87 \\
0,73\end{array}$ & $\begin{array}{l}0,86 \\
0,80\end{array}$ & $\begin{array}{l}0,87 \\
0,81\end{array}$ & $\begin{array}{l}0,87 \\
0,66\end{array}$ & $\begin{array}{l}0,86 \\
0,67\end{array}$ \\
\hline C-G & & & $\begin{array}{l}0,95 \\
0,95\end{array}$ & $\begin{array}{l}0,85 \\
0,84\end{array}$ & $\begin{array}{l}0,87 \\
0,86\end{array}$ & $\begin{array}{l}0,88 \\
0,85\end{array}$ & $\begin{array}{l}0,87 \\
0,86\end{array}$ \\
\hline $\begin{array}{l}\text { C-G } \\
\text { indexado }\end{array}$ & & & & $\begin{array}{l}0,94 \\
0,91\end{array}$ & $\begin{array}{l}0,95 \\
0,93\end{array}$ & $\begin{array}{l}0,96 \\
0,94\end{array}$ & $\begin{array}{l}0,95 \\
0,95\end{array}$ \\
\hline MDRD & & & & & $\begin{array}{l}0,99 \\
0,99\end{array}$ & $\begin{array}{l}0,99 \\
0,91\end{array}$ & $\begin{array}{l}0,99 \\
0,93\end{array}$ \\
\hline CKD-EPI & & & & & & $\begin{array}{l}0,99 \\
0,92\end{array}$ & $\begin{array}{l}0,99 \\
0,94\end{array}$ \\
\hline BIS-1 & & & & & & & $\begin{array}{l}0,99 \\
0,99\end{array}$ \\
\hline
\end{tabular}

Matriz que muestra el coeficiente de correlación lineal de Pearson (en cursiva) y el coeficiente de concordancia de Lin entre los diferentes métodos de estimación de la VFG en adultos mayores. Cl Cr: Clearance de creatinina de 24 h; Cl Cr corregido: Clearance de creatinina de $24 \mathrm{~h}$ normalizado a superficie corporal de 1,73 m²; C-G: Cockroft-Gault; C-G indexado: Cockroft-Gault normalizado a superficie corporal de 1,73 m²; MDRD: Modification of diet in renal disease; CKD-EPI: Chronic Kidney Disease Epidemiology Collaboration; BIS-1: Berlin Iniciative Study-1; FAS: Full-Age Spectrum.

Cockroft-Gault indexada, MDRD, CKD-EPI, BIS1 y FAS, siendo menor entre $\mathrm{ClCr}$ y ClCr corregido y el resto de los métodos (Tabla 5).

$\mathrm{Al}$ usar como referencia el $\mathrm{ClCr} / 24 \mathrm{~h}$ medido, todas las ecuaciones subestimaron la función renal, lo cual fue mayor para BIS-1 y FAS. Esta diferencia con el ClCr/24 h medido fue mayor para valores más altos de VFG (Figura 2).

\section{Discusión}

El valor del $\mathrm{ClCr} / 24 \mathrm{~h}$ medido sobrestima el valor de la VFG cuando esta es determinada con un método de referencia (Ej: clearance de inulina, iohexol, iothalamato, ${ }^{51} \mathrm{Cr}$-EDTA) en adultos mayores. Una explicación de ello es que el $\mathrm{ClCr} / 24 \mathrm{~h}$ no solo incluye la excreción de creatinina por filtración glomerular sino también la creatinina eliminada por secreción tubular. Esta última fracción aumenta cuando la VFG disminuye, como ocurre normalmente en adultos mayores y más aun, cuando ellos sufren una ERC ${ }^{11}$. En una comunicación que incluyó a 782 pacientes franceses con edades promedio de 72,8 $\pm 5,6$ años, $89 \%$ de los cuales tenía una ERC, el $\mathrm{ClCr} / 24 \mathrm{~h}$ medido fue $7,2 \mathrm{~mL} / \mathrm{min} / 1,73 \mathrm{~m}^{2}$ más alto que la VFG medida con ${ }^{51} \mathrm{Cr}-\mathrm{EDTA}{ }^{7}$.

Cuando el $\mathrm{ClCr} / 24 \mathrm{~h}$ no es medido, sino que es estimado con la fórmula de Cockcroft-Gault estandarizada por superficie corporal en adultos mayores, el valor obtenido uniformemente subestima el valor de la VFG medida con un método de referencia en hasta $21,5 \mathrm{~mL} / \mathrm{min} / 1,73 \mathrm{~m}^{2(7,12,13)}$, así como también subestima el $\mathrm{ClCr} / 24 \mathrm{~h}$ medido en una cuantía menor, entre 2 y $4 \mathrm{~mL} /$ $\min / 1,73 \mathrm{~m}^{2}{ }^{(14,15)}$ (Figura 1). En esta serie de adultos mayores la ecuación de Cockcroft-Gault subestimó el valor del $\mathrm{ClCr} / 24 \mathrm{~h}$ medido en 12,3 $\mathrm{mL} / \mathrm{min}$ (Tabla 2). Es por ello que se ha recomendado no utilizar la fórmula de Cockcroft-Gault en adultos mayores, porque aumenta artificialmente la prevalencia de ERC o clasifica a los enfermos en categorías más graves de ERC, además que en la generación de la ecuación en la década 1970-79 se utilizó un método de medición de la creatinina que actualmente no está vigente $e^{7,11}$. 


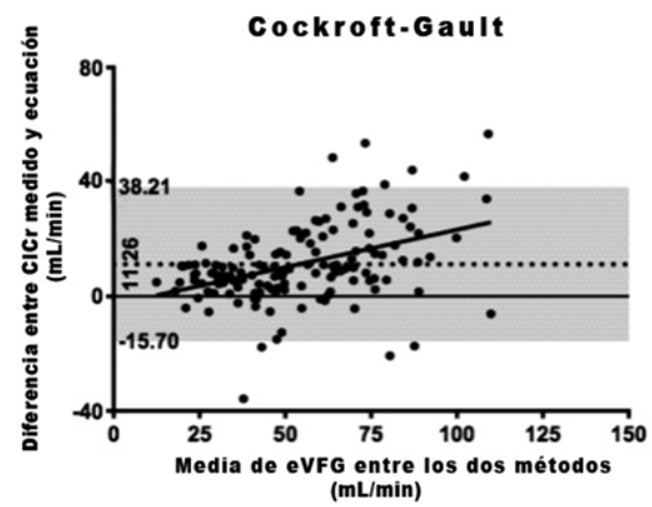

M D R D

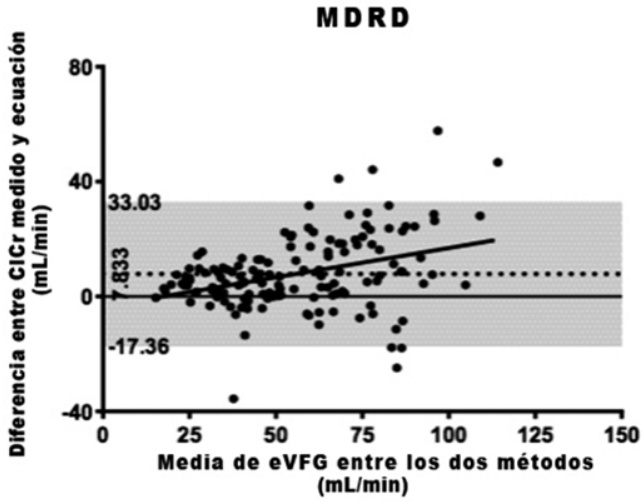

BIS-1

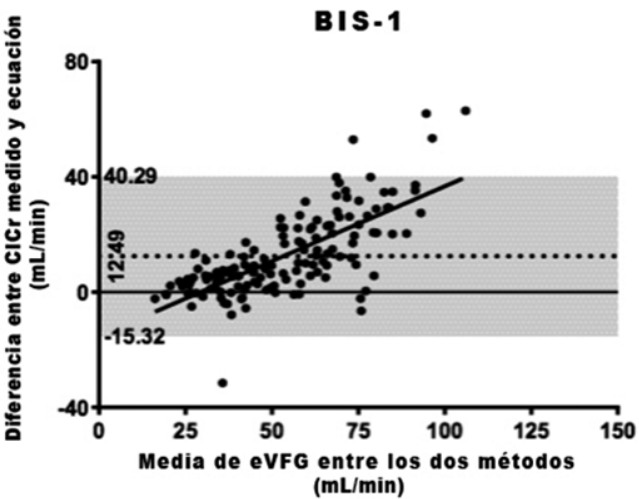

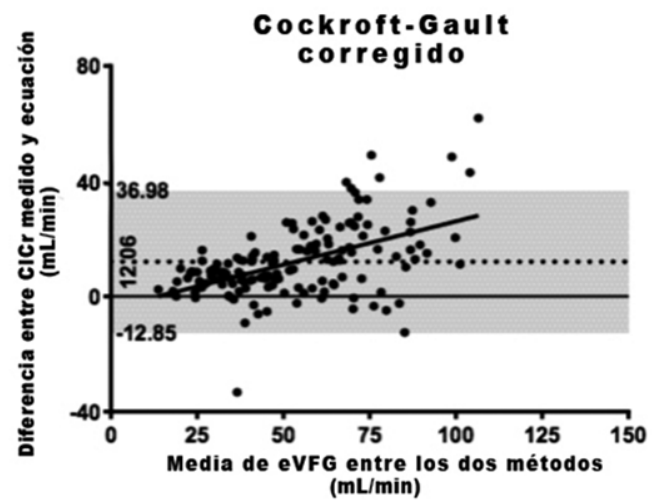

CKD-EP I

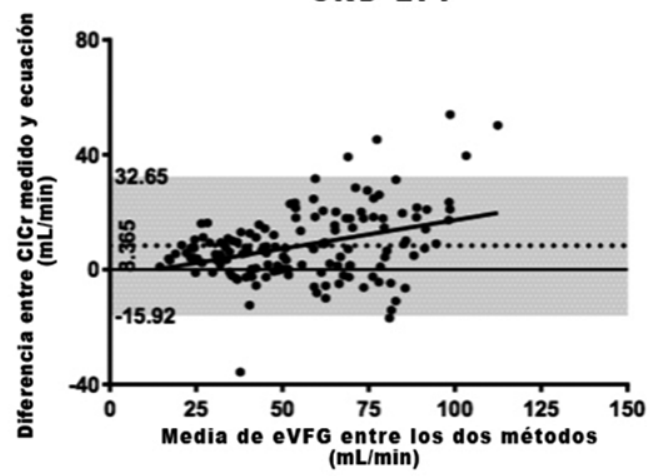

FAS

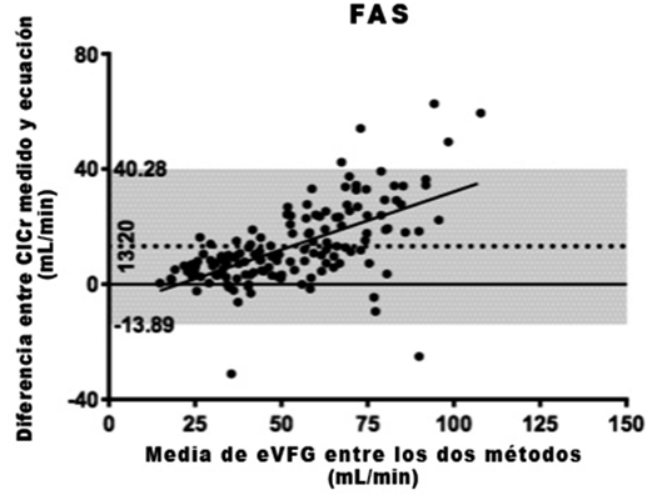

Figura 2. Gráficos de Bland-Altman para el clearance de creatinina corregido y otras fórmulas de estimación de la velocidad de filtración glomerular en adulto mayor. Cada gráfico de Bland-Altman muestra la relación entre la VFG estimada por el clearance de creatinina corregido y el estimado por las diferentes ecuaciones. Se muestra el promedio entre los dos métodos (eje $\mathrm{x}$ ) y la diferencia entre los 2 métodos (eje y). ClCr: Clearance de creatinina de 24 h; MDRD: Modification of diet in renal disease; CKD-EPI: Chronic Kidney Disease Epidemiology Collaboration; BIS-1: Berlin Iniciative Study-1; FAS: Full-Age Spectrum; eVFG: Velocidad de filtración glomerular estimada. 
Cuando se utiliza la fórmula MDRD para estimar la VFG en adultos mayores, habitualmente, hay una sobrestimación entre $6-7 \mathrm{~mL} / \mathrm{min} / 1,73 \mathrm{~m}^{2}$ respecto a los valores de VFG medidos con un método de referencia ${ }^{7,12,13}$. Ocurre lo mismo, generalmente, cuando se compara el valor obtenido con esta ecuación con el $\mathrm{ClCr} / 24 \mathrm{~h}$ medido, en que la diferencia puede llegar hasta los $19 \mathrm{~mL} /$ $\min / 1,73 \mathrm{~m}^{2(7,14,15)}$. En nuestra serie de adultos mayores, la ecuación mencionada subestimó el $\mathrm{ClCr} / 24 \mathrm{~h}$ medido en $7,8 \mathrm{~mL} / \mathrm{min} / 1,73 \mathrm{~m}^{2}$.

La fórmula CKD-EPI ha sido la actualmente recomendada por las guías KDIGO para estimar la VFG y para estadificar a los pacientes en las distintas categorías de $\mathrm{ERC}^{16}$. Esta ecuación, utilizada en pacientes mayores, habitualmente entrega valores similares a la VFG medida (mVFG) con alguno de los métodos de referencia. En 8 de 9 comunicaciones, la diferencia entre la eVFG y la mVFG fue inferior a 4,0 mL/min/1,73 $\mathrm{m}^{2(7,13,17-22)}$. En nuestra serie de adultos mayores, esta ecuación dio un valor menor en $8,4 \mathrm{~mL} / \mathrm{min} / 1,73 \mathrm{~m}^{2}$ respecto al $\mathrm{ClCr} / 24 \mathrm{~h}$ medido.

En esta serie de adultos mayores, los valores de la VFG estimada con las ecuaciones CKD-EPI y MDRD fueron casi idénticos (51,2 vs $51,8 \mathrm{~mL} /$ $\mathrm{min} / 1,73 \mathrm{~m}^{2}$ ). Algo muy parecido ocurrió en 9 series de adultos mayores en que la estimación de la VFG con ambas fórmulas en promedio no difirió en más de $2 \mathrm{~mL} / \mathrm{min} / 1,73 \mathrm{~m}^{2(7,13,17-20,23-25)}$.

Respecto a las ecuaciones desarrolladas para tener mayor validez en el grupo de adultos mayores, como BIS-1, las diferencias entre la mVFG con métodos de referencia y la estimada con esta fórmula en adultos mayores, en 5 series reportadas difiere en menos de $4,0 \mathrm{~mL} / \mathrm{min} / 1,73 \mathrm{~m}^{2}$, casi siempre subestimando la $\mathrm{mVFG}^{13,17,19,21,22,26}$. En nuestra serie, esta fórmula dio un valor de eVFG $12,5 \mathrm{~mL} / \mathrm{min} / 1,73 \mathrm{~m}^{2}$ más bajo que el $\mathrm{ClCr} / 24 \mathrm{~h}$ medido. Igual cosa sucedió con la fórmula FAS, en que por ser la más nueva hay poca información sobre su comportamiento en adultos mayores ${ }^{27}$. En nuestra serie difirió del $\mathrm{ClCr} / 24 \mathrm{~h}$ medido en $13,2 \mathrm{~mL} / \mathrm{min} / 1,73 \mathrm{~m}^{2}$.

En suma: La práctica de considerar en adultos mayores el resultado de la medición del $\mathrm{ClCr} / 24 \mathrm{~h}$ equivalente a la estimación de la VFG con las diversas fórmulas disponibles debe ser abandonada, por las amplias diferencias entre sus valores. Por los motivos previamente mencionados, deben preferirse las fórmulas CKD-EPI o las más nuevas como BIS-1 o FAS para estimar la VFG desechando las ecuaciones antiguas como Cockcroft-Gault y MDRD.

\section{Referencias}

1. Levey AS, Bosch JP, Lewis JB, Greene T, Rogers N, Roth D. A more accurate method to estimate glomerular filtration rate from serum creatinine: a new prediction equation. Modification of diet in renal disease study group. Ann Intern Med. 1999; 130: 461-70.

2. Levey AS, Coresh J, Green T, Stevens LA, Zhang YL, Hendricksen S, et al. Using standardized serum creatinine values in the modification of diet in renal disease study equation for estimating glomerular filtration rate. Ann Intern Med. 2006; 145: 247-54.

3. Levey AS, Stevens LA, Schmid CH, Zhang YL, Castro AF, Feldman HI, et al. A new equation to estimate glomerular filtration rate. Ann Intern Med. 2009; 150: 604-12.

4. Pottel H, Hoste L, Dubourg L, Ebert N, Schaeffner E, Eriksen BO, et al. An estimated glomerular filtration rate equation for the full age spectrum. Nephrol Dial Transplant. 2016; 31: 798-806.

5. Schaeffner ES, Ebert N, Delanaye P, Frei U, Gaedeke J, Jacob O, et al. Two novel equations to estimate kidney function in persons aged 70 years or older. Ann Intern Med. 2012; 157: 471-81.

6. Cockcroft DW, Gault MH. Prediction of creatinine clearance from serum creatinine. Nephron. 1976; 16: 31-41.

7. Flamant M, Haymann JP, Vidal-Petiot E, Letavernier E, Clerici C, Boffa JJ, et al. GFR estimation using the Cockcroft-Gault, MDRD study, and CKD-EPI equations in the elderly. Am J Kidney Dis. 2012; 60: 847-9.

8. Wetzels JF, Kiemeney LA, Swinkels DW, Willems HL, den Heijer M. Age - and gender-specific reference values of estimated GFR in caucasians: the Nijmejen biomedical study. Kidney Int. 2007; 72: 632-7.

9. Glassock RJ, Winearls C. An epidemic chronic kidney disease: fact or fiction? Nephrol Dial Transplant. 2008; 23: 1117-21.

10. Mayo Clinic, Mayo Medical Laboratories, Creatinine, 24 hour, urine, https://www.mayomedicallaboratories.com/ test-catalog/Clinical+and+Interpretative/8513(2018) accedido el 13 de agosto 2020.

11. Garasto S, Fusco S, Corica F, Rosignuolo M, Marino A, Montesanto A, et al. Estimating glomerular filtration rate in older people. Biomed Res Int. 2014; 2014: 916542 . 
12. Fehrman-Ekholm I, Skeppholm L. Renal function in the elderly ( $>70$ years old) measured by means of iohexol clearance, serum creatinine, serum urea and estimated clearance. Scand J Urol Nephrol. 2004; 38; 73-7.

13. Changjie G, Xusheng Z, Feng H, Shuguang Q, Jianwen L. Evaluation of glomerular filtration rate by different equations in chinese elderly with chronic kidney disease. Int Urol Nephrol. 2017; 49: 133-41.

14. Péquignot R, Belmin J, Chauvelier S, Gauvert JY, Konrat $\mathrm{C}$, Duron E, et al. Renal function in older hospital patients is more accurately estimated using the Cockcroft-Gault formula than the modification diet in renal disease formula. J Am Geriatr Soc. 2009; 57: 1638-43.

15. Gómez-Pavón J, Gálvez Barrón C, Baztán Cortés JJ, Ruipérez Cantera I. Comparación del uso de las ecuaciones de estimación del filtrado glomerular renal en personas de 75 años o más sin enfermedad renal conocida. Med Clin. (Barc) 2010; 134: 346-9.

16. KDIGO Gorostidi M, Santamaría R, Alcázar R, Fernández-Fresnedo $\mathrm{G}$, Galcerán JM, Goicochea $\mathrm{M}$, et al. Documento de la sociedad española de nefrología sobre las guías KDIGO para la evaluación y el tratamiento de la enfermedad renal crónica. Nefrología 2014; 34 (3): 302-16.

17. Koppe L, Klich A, Dubourg L, Ecochard R, Hadj-Aissa A. Performance of creatinine-based equations compared in older patients. J Nephrol. 2013; 26: 716-23.

18. Kilbride HS, Stevens PE, Eaglestone G, Knight S, Carter JL, Delaney MP, et al. Accuracy of the MDRD (Modification of diet in renal disease study) and CKD-EPI (CKD epidemiology collaboration) equations for estimation of GFR in the elderly. Am J Kidney Dis. 2013; 61: 57-66.

19. Lopes MB, Araújo LQ, Passos MT, Nishida SK, Kirsztajn GM, Cendoroglo MS, et al. Estimation of glomerular filtration rate from serum creatinine and cystatin $\mathrm{C}$ in octogenarians and nonagenarians. BMC Nephrol. 2013; 14: 265.

20. Zhu Y, Ye X, Zhu B, Pei X, Wei L, Wu J, et al. Compari- sons between the 2012 new CKD-EPI (Chronic kidney disease epidemiology collaboration) equations and other four approved equations. PloS One 2014; 9: e84688

21. Vidal-Petiot E, Haymann JP, Letavernier E, Serrano F, Clerici C, Boffa JJ, et al. External validation of the BIS (Berlin Initiative Study)-1 GFR estimating equation in the elderly. Am J Kidney Dis. 2014; 63: 859-67.

22. Alshaer IM, Kilbride HS, Stevens PE, Eaglestone G, Knight S, Carter JL, et al. External validation of the Berlin equations for estimation of GFR in the elderly. Am J Kidney Dis. 2014; 63: 859-67.

23. Bustos-Guadaño F, Martín-Calderón JL, Criado-Álvarez JJ, Muñoz-Jara R, Cantalejo-Gutiérrez A, Mena-Moreno MC. Estimation of the glomerular filtration rate in people older than 85: comparisons between CKD-EPI, MDRD-IDMS and BIS1 equations. Nefrología 2017; 37: 172-180.

24. Willems JM, Vlasveld T, den Elzen WP, Westendorp RG, Rabelink TJ, de Craen AJ, et al. Performance of Cockcroft-Gault, MDRD, and CKD-EPI in estimating prevalence of renal function and predicting survival in the oldest old. BMC Geriatr. 2013; 13: 113.

25. Salvador-González B, Rodríguez-Latre LM, Güell-Miró R, Álvarez-Funes V, Sanz-Ródenas H, Tovillas-Morán FJ. Estimación del filtrado glomerular según MDRD-4 IDMS y CKD-EPI en individuos de edad igual o superior a 60 años en atención primaria. Nefrología 2013; 33: 552-63.

26. Corsonello A, Roller-Wirnsberger R, Wirnsberger G, Arnlov J, Carlsson AC, Tap L, et al. Clinical implications of estimating glomerular filtration rate with three different equations among older people. Preliminary results of the Project "Screening for chronic kidney disease among older people across Europe (SCOPE)". J Clin Med 2020; 9: 294.

27. Pottel H, Delanaye P, Schaeffner E, Dubourg L, Eriksen BO, Melsom T, et al. Estimating glomerular filtration rate for the full age spectrum from serum creatinine and cystatin C. Nephrol Dial Transplant 2017; 32: 497-507. 